\title{
CONTINUA OF MINIMUM CAPACITY ${ }^{1}$
}

G. C. EVANS

1. Surfaces containing a given volume. In an endeavour to simplify a proof of Liapounoff [2], to the effect that in the problem of the forms of equilibrium of rotating liquids the sphere would be the only form for a liquid at rest, Poincaré [1] was led to the consideration of electric capacities of solids of given volume, and arrived at the result that among such bodies the sphere would have minimum capacity. The present paper originated in the question of the determination of the surface sheet, without volume, which would be bounded by a given closed curve in space, and, among all such surfaces, have minimum capacity.

In the discussion of his problem, Poincaré assumes tacitly that there do exist one or more bodies of the given volume, with smooth boundaries, which furnish relative minima for the capacity with respect to neighboring forms; and his treatment amounts to a proof that among these the sphere furnishes the absolute minimum.

Let the body $F$, with smooth exterior boundary $S$, be considered as a conductor on which a positive charge $m$ is spread so as to be in equilibrium-that is, the charge lies entirely on the surface $S$ with a surface density $\sigma(P)$, and its potential $V(M)$ has a constant value $V_{0}$ within $S$, is continuous across $S$, satisfies Laplace's equation $\nabla^{2} V=0$ outside $S$ and vanishes at infinity. The density on $S$ is given by the equation

$$
\sigma(P)=-\frac{1}{4 \pi} \frac{d V}{d n}, \quad n \text { the exterior normal. }
$$

The energy of the distribution may be written in the equivalent forms

(1) $I=\frac{1}{8 \pi} \int_{W}(\nabla V)^{2} d P=\frac{1}{8 \pi} \int_{W}\left(V_{x}^{2}+V_{y}^{2}+V_{z}^{2}\right) d P, W=$ entire space,

(2) $I=\frac{1}{2} \int_{S} \sigma(P) V_{0} d P=\frac{1}{2} m V_{0}$.

The capacity of $K$ of $F$ is defined by the equation

$$
K V_{0}=m \quad \text { or } \quad I=\frac{m^{2}}{2 K}=\frac{1}{2} K V_{0}^{2} .
$$

${ }^{1}$ Presidential address presented to the Society, January 1, 1941, under the title Surfaces of minimum capacity. 
According to Poincaré, the variation $\delta I$ of $I$ due to a displacement of the points of $S$, along normals, of amount $\delta n(P)=\epsilon \psi(P)$ is given by the equation

$$
\delta I=-\frac{1}{8 \pi} \int_{S} \delta n(P)(\nabla V)^{2} d P=-\frac{\epsilon}{8 \pi} \int_{S} \psi(P)\left(\frac{d V}{d n}\right)^{2} d P,
$$

the integral of the variation of the function $V(P)$ itself being negligible since the conductor distribution is in equilibrium. That (4) thus follows from (1) is not altogether evident. It may be justified, however, in the following way.

Let $S_{1}$ be the displaced surface, $P_{1}, Q_{1}$ points on $S_{1}$ corresponding to points $P, Q$ on $S$. Since for a given total mass $m$ on $S$ the energy for a conductor distribution is a minimum, $I$ will have a stationary value for small changes of the distribution $m(e)$ of $m$, and we may neglect such changes. Hence we may assume the relation

$$
\Delta I=\frac{1}{2} \int_{S} d m\left(e_{Q}\right) \int_{S}\left(\frac{1}{P_{1} Q_{1}}-\frac{1}{P Q}\right) d m\left(e_{P}\right),
$$

the potential at an arbitrary point $M$ being given by the formula

$$
V(M)=\int_{S} \frac{\sigma(P)}{M P} d P=\int_{S} \frac{d m\left(e_{P}\right)}{M P} .
$$

We substitute

$$
\frac{1}{P_{1} Q_{1}}-\frac{1}{P Q}=\left(\frac{1}{P_{1} Q_{1}}-\frac{1}{P_{1} Q}\right)+\left(\frac{1}{P_{1} Q}-\frac{1}{P Q}\right)
$$

and change the order of integration in the integral of this second parenthesis, at the same time interchanging $P$ with $Q$ and $P_{1}$ with $Q_{1}$ so that we shall have

$$
\Delta I=\frac{1}{2} \int_{S} \sigma(Q) d Q \int_{S}\left\{\left(\frac{1}{P_{1} Q_{1}}-\frac{1}{P_{1} Q}\right)+\left(\frac{1}{P Q_{1}}-\frac{1}{P Q}\right)\right\} \sigma(P) d P .
$$

We denote by $V_{1}\left(Q^{\prime}\right)$ the potential of the displaced mass at some point $Q^{\prime}$ between $Q$ and $Q_{1}$, and by $V\left(Q^{\prime \prime}\right)$ the potential of the original mass at some point $Q^{\prime \prime}$ between $Q$ and $Q_{1}$. Then

$$
\Delta I=\frac{\epsilon}{2} \int_{S} \sigma(Q) \psi(Q)\left\{\left(\frac{d V_{1}}{d n}\right)_{Q^{\prime}}+\left(\frac{d V}{d n}\right)_{Q^{\prime \prime}}\right\} d Q .
$$

We assume that $S$ and $S_{1}$ are sufficiently smooth so that $\sigma(Q)$ is bounded and so that the derivatives of the potential approach the 
limits given by the usual well known formulae, as $\epsilon$ tends to zero. Then

$$
\begin{aligned}
& \lim _{\epsilon \rightarrow 0}\left(\frac{d V}{d n}\right)_{Q^{\prime \prime}}=\mp 2 \pi \sigma(Q)+\int_{S} \frac{\cos \left(n_{Q}, Q P\right)}{P Q^{2}} \sigma(P) d P, \\
& \lim _{\epsilon \rightarrow 0}\left(\frac{d V_{1}}{d n}\right)_{Q^{\prime}}= \pm 2 \pi \sigma(Q)+\int_{S} \frac{\cos \left(n_{Q}, Q P\right)}{P Q^{2}} \sigma(P) d P,
\end{aligned}
$$

the upper sign being employed when $\psi(Q)>0$, the lower when $\psi(Q)<0$. Hence, for $\delta I=\epsilon \lim _{\epsilon \rightarrow 0}(\Delta I / \epsilon)$, we have the value

$$
\delta I=\epsilon \int_{S} \sigma(Q) \psi(Q) d Q \int_{S} \frac{\cos \left(n_{Q}, Q P\right)}{P Q^{2}} \sigma(P) d P .
$$

Inside the conductor the potential is constant, so that as a point approaches $S$, from inside, the value $d V / d n_{-}$is zero. Accordingly, by combining the first two of equations (5), we have

$$
\frac{d V}{d n_{+}}=-4 \pi \sigma(Q)=2 \int_{S} \frac{\cos \left(n_{Q}, Q P\right)}{P Q^{2}} \sigma(P) d P .
$$

Thus the formula (6) for $\delta I$ reduces to the value given by (4).

In order that the capacity be a minimum, with given volume, it is necessary that $\delta I=0$, where $\delta I$ is given by $(4)$ and $\psi(P)$ is subject to the condition

$$
\int_{S} \psi(P) d P=0
$$

It follows therefore that $\left(d V / d n_{+}\right)^{2}$ is constant on $S$, and since $d V / d n_{+}=-4 \pi \sigma$, we have

$$
\sigma(P)=\text { const. on } S .
$$

Moreover, since now $\sigma$ is constant on $S$, we may write the formula for $\delta I$ in the form (the total mass $m$ of course remaining unchanged)

$$
\delta I=-\frac{S^{2} \sigma^{2}}{2 K^{2}} d K=-2 \pi \sigma^{2} d \tau
$$

in which the volume is changed, and $d \tau$ and $d K$ are corresponding differential changes of volume and capacity. For such changes, then,

$$
\frac{S^{2} d K}{K^{2}}=4 \pi d \tau
$$


Suppose now that the body is deformed so as to remain similar to itself. Its capacity will change in the ratio of corresponding lengths, so that

$$
\frac{d K}{K}=\frac{1}{3} \frac{d \tau}{\tau},
$$

and, in the case of bodies for which $(\tau)$ is satisfied,

$$
K=\frac{1}{12 \pi} \frac{S^{2}}{\tau}
$$

The sphere satisfies the condition (7), and it has the smallest $S$ for a given $\tau$. Hence if there is any smooth $S$ which has the minimum capacity for a given volume, the sphere is that body.

Incidentally, we notice that if we make no requirement about the volume, but consider the surface merely as a sheet or cap, bounded, for instance, by a given closed curve in space, the requirement that the capacity be a minimum implies, if $S$ be sufficiently smooth, that $\delta I$ be zero, as given by (6). Since there is no restriction on $\psi(Q)$ in the neighborhood of any point of $S$, not on the boundary, we have the relation

$$
\int_{S} \frac{\cos \left(n_{Q}, Q P\right)}{P Q^{2}} \sigma(P) d P=0=\int_{S} \frac{\cos \left(n_{Q}, Q P\right)}{P Q^{2}} d m\left(e_{P}\right) .
$$

By means of the first of (5) the equation (8) may be written in the form

$$
\frac{d V}{d n_{+}}+\frac{d V}{d n_{-}}=0
$$

where, of course, we still have the relation

$$
\frac{d V}{d n_{+}}-\frac{d V}{d n_{-}}=-4 \pi \sigma \text {. }
$$

2. Capacity and logarithmic potential. In the discussion of the stability of the form of a rotating liquid, and in particular the spherical form of a liquid at rest, Liapounoff treated the second variation of the energy integral and showed it to be positive. Poincaré endeavored, as we have mentioned, to provide a simpler treatment for this special case. But in order to complete the problem in a satisfactory manner without assuming the existence of a smooth form for which capacity is a minimum further details are necessary. The possibility of a fuller 
use of fundamental inequalities for this purpose was noticed by Faber [3] and Szegö [5]. This use is sufficiently illustrated in some corresponding, but perhaps more difficult, problems in the plane, and accordingly we turn to those.

For the plane, as in space, the capacity is to be regarded as an index of the charge which is necessary in order to raise the body to a given constant potential $V_{0}$. The equation $V_{0}=m / K$, however, if carried over to the logarithmic potential has the disadvantage of yielding infinite and negative capacities. A more satisfactory definition, especially from the point of view of the complex variable, is given by

$$
V_{0}=m \log \frac{1}{K} ; \quad K=e^{-V_{0} / m} .
$$

This is the one which we adopt. It is equivalent to the "transfinite diameter." By means of it, capacity is of dimension one in length in the plane (as in space), with regard to transformations of similarity, and the capacity of a circle (like that of a sphere) is equal to its radius.

Given an arbitrary bounded closed set $F$ in the plane it may not be possible to distribute the mass $m$ on $F$ in such a way that the potential will remain constant everywhere on $F$ because the frontier $s$ which bounds the infinite domain $T$ exterior to $F$ may not consist entirely of points which are regular, with respect to $T$, for the Dirichlet problem with continuously given boundary values on $s$-for example, if $s$ has isolated points. We can however approximate to $T$ by a nested sequence of domains the boundary of each of which consists of a finite number of closed simple analytic curves, and thus define $K$ by the obvious limiting process. In particular, if for a given $m$ the potential $V_{0}$ does not remain bounded in the limit, the capacity of $F$ is zero.

Accordingly we may limit ourselves to the sets $F$ whose exterior frontiers consist only of points regular with respect to the Dirichlet problem. Let $u(M)$ be the potential of unit mass on $F$ distributed so as to have constant potential $V_{0}$ on $F$. Then, with $r=O M$ where $O$ is any fixed point,

$$
u(M)=\log \frac{1}{r}+H(M)=-\log r+H(M)
$$

with $H(M)$ a function which is harmonic in $T$ and vanishes at infinity. Consequently the function (a special case of the Green's function)

$$
V(M)=V_{0}-u(M),
$$

becomes infinite like $\log r$ at $\infty$ and vanishes on $F$. In fact, 


$$
V(M)=\log r-G(M), \quad G(M)=H(M)-V_{0},
$$

where $G(M)$ is harmonic in $T$, and regular at $\infty$, at which point it takes on the value $G(\infty)=-V_{0}$. We have therefore

$$
K=e^{G(\infty)} .
$$

We follow now the procedure as sketched briefly by Szegö for analogous problems [5]. Consider the level curves $V(M)=\lambda$ of the function $V(M)$. For $\lambda>0$ these curves have no points in common with $F$ since the points of the boundary $s$ are regular; except for a finite number of values of $\lambda$, provided that $\lambda$ is bounded away from 0 , the locus $V(M)=\lambda$ consists of a finite number of simple closed analytic curves $[6, b]$, and for large values of $\lambda$ of a single piece. We denote this locus (and also its total length) by $C_{\lambda}$, the total area inside it by $A_{\lambda}$ and the exterior normal direction by $n$. Thus $\lim _{\lambda \rightarrow 0} A_{\lambda}=$ meas. $F$.

We have

$$
2 \pi=\int_{c_{\lambda}} \frac{d V}{d n} d s, \quad \frac{d A_{\lambda}}{d \lambda}=\int_{C_{\lambda}} \frac{d n}{d V} d s,
$$

the first equality being an expression of the fact that $V$ is harmonic except for the additive term $\log r$. But by Schwarz's inequality and the isoperimetric inequality applied successively, we have

$$
\begin{aligned}
2 \pi \frac{d A_{\lambda}}{d \lambda} & =\int_{C_{\lambda}}\left(\left(\frac{d V}{d n}\right)^{1 / 2}\right)^{2} d s \cdot \int_{C_{\lambda}}\left(\left(\frac{d n}{d V}\right)^{1 / 2}\right)^{2} d s \\
& \geqq\left(\int_{C_{\lambda}} d s\right)^{2}=C_{\lambda}^{2} \geqq 4 \pi A_{\lambda} .
\end{aligned}
$$

Thus $(1 / A) d A / d \lambda \geqq 2$, and

$$
\log A_{\lambda}-\log A_{\lambda_{1}} \geqq 2\left(\lambda-\lambda_{1}\right) .
$$

Hence if we let $\lambda_{1} \rightarrow 0$, we have

$$
A_{\lambda} \geqq(\text { meas. } F) e^{2 \lambda} .
$$

In this expression we now let $\lambda \rightarrow \infty$. For large values of $\lambda$ we may use polar coordinates, and write, since $r=e^{\lambda} e^{G(M)}$,

$$
A_{\lambda}=\frac{1}{2} \int_{0}^{2 \pi} r^{2} d \theta=\frac{1}{2} e^{2 \lambda} \int_{0}^{2 \pi} e^{2 G(M)} d \theta .
$$

Consequently 


$$
\frac{1}{2} \int_{0}^{2 \pi} e^{2 G(M)} d \theta \geqq \text { meas. } F ;
$$

and if we let $\lambda \rightarrow \infty$ so that $G(M) \rightarrow G(\infty)$, we shall have

$$
\lim _{\lambda \rightarrow \infty} \int_{0}^{2 \pi} e^{2 G(M)} d \theta=2 \pi K^{2}
$$

Hence

$$
\pi K^{2} \geqq \text { meas. } F \text {. }
$$

The equality sign in (12) can hold only if it holds identically in $\lambda$; that is, by Schwarz's inequality, only if $d V / d n$ and $d n / d V$ are proportional on each $C_{\lambda}$ so that $d n / d V$ is a function of $\lambda$ and the $C_{\lambda}$ form a set of parallel curves, and by the isoperimetric inequality, only if the $C_{\lambda}$ are circles. In this case $F$ is a circle and its radius is $K$. Since the difference of the two sides of (13) represents the integral from 0 to $\infty$ of a not negative quantity, the same result holds for the equality sign in (13).

The inequality (13) holds for any bounded closed set $F$. In fact, both sides of the inequality are obtained by the same process in terms of a nested sequence of approximate domains. But other sets besides the circle, if $F$ is not a single continuum, satisfy the relation $\pi K^{2}=F$.

3. Applications of conformal mapping. Except for a rotation and translation, a conformal transformation which maps the exterior of a bounded continuum $F$ in the $z$ plane in to the exterior of a circle of radius $r$ in the $w$ plane, without distortion at $\infty$, may be written in the form

$$
z-w=\sum_{1}^{\infty} \alpha_{n} w^{-n}
$$

The radius $r$ is called the Robin constant. We find that $r=K$, where $K$ is the capacity of $F$. This is seen immediately by comparing (14) with the transformation

$$
w=r e^{V(z)+i U(z)}
$$

where $U(z)=U(M)$ is the function conjugate to $V(z)=V(M)$, and by noting the value of $|d w / d z|$ in (15) at $\infty$.

A direct calculation from (14) in this case shows that

$$
\text { meas. } F=\pi r^{2}-\pi \sum_{1}^{\infty} n r^{-2 n}\left|\alpha_{n}\right|^{2}
$$


so that in particular meas. $F \leqq \pi r^{2}$, which is a special case of (13), and

$$
\left|\alpha_{1}\right| \leqq r^{2} \text {. }
$$

On the other hand, if $A$ is an interior domain in the $Z$ plane, containing the origin, which is mapped conformally on the interior of a circle $|W|=R$ so that $Z=0$ goes into $W=0$ and there is no distortion at $Z=0$, the transformation, except for a possible rotation, may be written in the form

$$
Z=W+\sum_{2}^{\infty} \beta_{n} W^{n}
$$

Hence

$$
A \geqq \pi R^{2}+\pi \sum_{2}^{\infty} n\left|\beta_{n}\right|^{2} R^{2 n} \geqq \pi R^{2} .
$$

By means of the transformations

$$
w=\frac{1}{W}, \quad z=\frac{1}{Z}
$$

the situation is reduced to a transformation of exterior domains, in which $r=1 / R$. But the transformation of these exterior domains is given by

$$
\begin{aligned}
z & =\left(\frac{1}{w}+\sum_{2}^{\infty} \beta_{n} w^{-n}\right)^{-1}=w\left(1+\beta_{2} w^{-1}+\beta_{3} w^{-2}+\cdots\right)^{-1} \\
& =w\left(1-\beta_{2} w^{-1}+\cdots\right) .
\end{aligned}
$$

Consideration of the resulting univalent relation between branches of $z^{\prime}=z^{1 / 2}$ and $w^{\prime}=w^{1 / 2}$ yields immediately, by (16), the relation

$$
\left|\beta_{2}\right| \leqq \frac{2}{R} \text {. }
$$

The above results, which are involved in a direct comparison of the coefficients of the power series are of course well known. But also other known results are obtained in terms of them by means of elementary statements about capacity. From the definition of capacity and the fact that $V_{0}$ is the potential due to a distribution of positive mass, there follows easily the statement that if $F_{1}$ is contained in $F_{2}$, or is the orthogonal projection of $F_{2}$ on a line, then $K\left(F_{1}\right) \leqq K\left(F_{2}\right)$. By means of the conformal transformation ${ }^{2}$

${ }^{2}$ This is the base transformation for the study of aerofoils. 


$$
w=\frac{1}{2}\left(z+\left(z^{2}-a^{2}\right)^{1 / 2}\right)
$$

in which $w=(1 / 2)\left(x+\left(x^{2}-a^{2}\right)^{1 / 2}\right)$ when $z=x$ is real and $>a$, the region exterior to the segment $(-a, 0),(a, 0)$ is transformed into the exterior of the circle $|w|=a / 2$, without distortion when $z=\infty$. Hence the capacity of a line segment of length $2 a$ is $a / 2$. Moreover the capacity of a segment of a straight line of length $l$ is less than that of a collection of segments of the line of the same total length $l$.

Let $p$ be the projection, of linear measure $l$; on an arbitrary straight line, of the closed bounded set $F$. Then $K(p) \geqq l / 4$ and $K(p) \leqq K(F)$. Hence

$$
l \leqq 4 K(F) .
$$

The theorem expressed by (19) was proved in slightly less general form by Pólya, by the application of Tchebytchef polynomials in the definition of transfinite diameter [4].

Consider again an interior domain and the transformation (17). Let $d$ be the minimum distance from $O$ to a point of the boundary of $A, R$ the radius of the corresponding circle in the $W$ plane, there being again no distortion at the origins, which correspond. We have immediately the Koebe result: $d \geqq R / 4$. In fact, there is a point $P$ on the boundary of $A$ such that $O P=d$. Its image $P^{\prime}$ is a point of $F$, where $F$ is the complement of the infinite domain obtained from $A$ by the transformation $z=Z^{-1}$, and the capacity $K(F)$ is the radius $r=1 / R$ of the corresponding circle in the $w$ plane, with $w=W^{-1}$. But, denoting $O P^{\prime}$ by $d^{\prime}$, we have from (19)

$$
\frac{1}{d}=d^{\prime} \leqq 4 K(F)=4 r=\frac{4}{R},
$$

from which the Koebe result follows. That $R / 4$ is the best result obtainable follows from the consideration of a region in the $z$ plane which is a narrow approximation to a straight segment.

Again, of all the circles $C$ contained in $A$ and containing $O$, the radii have an upper bound $l$, and there is a circle of radius $l$, whose interior lies in $A$ and such that $O$ lies in the circle or on its circumference. If this situation is interpreted in terms of the transformations $z=Z^{-1}, w=W^{-1}$ we find a circle $C^{\prime}$, of radius $l^{\prime}$, circumscribed about $F$, but not necessarily the smallest one.

The smallest circle $C^{\prime \prime}$ which circumscribes $F$ has a radius $l^{\prime \prime}$ which is $\leqq \eta / 3^{1 / 2}$, where $\eta$ is the diameter of $F$. But $\eta \leqq 4 K(F)$, so that $l^{\prime \prime} \leqq 4 r / 3^{1 / 2}$. The image of $C^{\prime \prime}$ in the $z$ plane is a circle $C$ in $A$ and containing $O$ in its interior. Let $P, Q$ be opposite ends of a diameter of $C$ 
through $O$. Then $P^{\prime}, Q^{\prime}$ are opposite ends of a diameter of $C^{\prime \prime}$ through $O$, and

$$
l \geqq \frac{1}{2}(P O+O Q)=\frac{1}{2}\left(\frac{1}{P^{\prime} O}+\frac{1}{O Q^{\prime}}\right) \geqq \frac{1}{l^{\prime \prime}},
$$

since the minimum value of $\left(1 / P^{\prime} O\right)+\left(1 / O Q^{\prime}\right)$ is obtained when $P^{\prime} O=O Q^{\prime}$. Accordingly

$$
l \geqq \frac{3^{1 / 2}}{4 r}=\frac{3^{1 / 2}}{4} R .
$$

The value $.43 R$ is not, however, the best value which can be obtained for the lower bound of $l$. By means of stricter inequalities on the coefficients of the Taylor series R. M. Robinson obtains the value $\frac{1}{2} R$. And if the requirement that $O$ shall be contained in the circle is dropped, the radius of the largest circle which lies in $A$ is at least as great as a number $k$, which as Landau shows [7] satisfies the inequality $k \geqq .55 R$, and as Robinson shows [8] satisfies also the inequality $k \leqq .66 R$.

4. Open curves of minimum capacity. The statement (19) about the capacity of the projection of $F$ may be reworded as follows:

Of all continua which contain two fixed points $A, B$, the straight segment $A B$ has the minimum capacity.

This theorem involves a new orientation towards the question, for it suggests the discussion of the continuum of minimum capacity which contains a given arbitrary collection of fixed points, finite in number. Pólya has considered this problem [4] and obtained the solution of it in the case of $2 n$ points spaced regularly, $n$ on each of two concentric circumferences, the desired continuum consisting in this case of the radial lines drawn from the points to the center of the circle.

The situation for $n$ arbitrary points is discussed by Grötsch $[9, \mathrm{~b}]$. The aspects of the problem which are involved are seen in the case of three points in the $z$ plane. Consider a circumference of radius $r$ in the $w$ plane, and on it three points $w_{1}=r+0 i, w_{2}=r e^{\left(\phi_{1}+\phi_{2}\right) i}$, $w_{3}=r e^{-\left(\phi_{1}+\phi_{3}\right) i}$ with $\phi_{1}, \phi_{2}, \phi_{3}$ positive angles of sum $\pi$. A conformal transformation, with unit magnification at $\infty$, of the exterior of the unit circle into the exterior of some continuum $C$ in the $z$ plane exists, in which pairs of symmetrical points on the arcs $\left[0, \phi_{1}\right],\left[0,-\phi_{1}\right]$, respectively, adjacent to $w_{1}$, go into single points of the $Z$ plane; and similarly for the arcs adjacent to $w_{2}$ and $w_{3}$. The three points $r e^{i \phi_{1}}$, $r e^{-i \phi_{1}}, r e^{i\left(\phi_{1}+2 \phi_{2}\right)}$ thus go into one point $z^{\prime}$, and the points $w_{1}, w_{2}, w_{3}$ 
correspond to certain distinct non-collinear points $z_{1}, z_{2}, z_{3}$. In fact, collinear points $z_{1}, z_{2}, z_{3}$ would arise only if one of the arcs $\phi_{1}, \phi_{2}, \phi_{3}$ were zero, as is suggested by (18). The continuum $C$ thus consists of three analytic rays from $z^{\prime}$ to $z_{1}, z_{2}, z_{3}$.

It is proved that $C$ has the least capacity of any continuum which contains the three points $z_{1}, z_{2}, z_{3}$ regarded as fixed, by reducing the question to the consideration of the maximum sum of "modular ratios" for an area $[9$, a]. This number is in fact equal to the upper bound of the Dirichlet integral of a function $u$ summed over strips into which an area is subdivided, where at the ends of the strips $u$ has the values 0 and 1 respectively, and along the sides of the strips $d u / d n=0$, and $u$ satisfies Laplace's equation in each strip. It is then shown by examination of the parameters involved in the transformation, with reference to a principle of continuity in the variation of those parameters, that the above treatment is equivalent to that of the general case. The situation with $n$ points is treated in the same manner, except that intermediate arcs, not abutting on any $w_{i}$, arise from the restrictive nature of the conformal transformation. ${ }^{3}$

5. Open surfaces of minimum capacity. In three dimensions, the simplest problem analogous to those just treated would be to prove the existence of a surface sheet, bounded by a given closed curve in space, which among all such surfaces would have minimum capacity $[10, a, b]$. This problem would be the counterpart of the simplest plane problem, namely, the continuum of minimum capacity containing two given points. But it may be of interest for the record that the author put to himself and solved the space problem first, before becoming familiar with the problem in the plane, and the latter has been introduced in this address, with its fundamental relation to conformal representation, mostly as an extension of the space problem rather than the reverse. In fact, it will be seen later that the statements of the two problems do not exactly correspond.

We consider a simple closed space curve $s$, itself of zero capacity, in our three-dimensional euclidean space $B$ and topologically equivalent to a circle in the sense that there exists a one-one continuous transformation $\xi$ of a sufficiently large sphere containing $s$ into itself, in such a way that $s$ goes into a circle $\sigma$ interior to the sphere. For convenience, we suppose that the points of the surface of the sphere are unchanged by the transformation, and we may regard $\xi$ as extended outside the sphere as the identical transformation.

\footnotetext{
${ }^{3}$ The author apologizes for the skimpy treatment of Grötsch's important contribution. Lack of space requires him to refer the reader to the cited memoirs.
} 
Let now $S$ be a piece of surface consisting of a closed set of points which contains $s$ and which, except in the neighborhood of $s$, consists of a finite number of regular surface elements, each surface element having bounded curvatures. Then, without specifying further for the moment the notion of $s$ being the boundary of $S$, the analysis of $\$ 1$ applies, and at every point in a surface element, not on an edge of the element or on $s$, the conductor potential of $S$ will satisfy the relation $\left(8^{\prime}\right)$. In order, however, not to have to distinguish between local positive and negative normal directions at points of $S$ we shall consider normal directions $n_{1}, n_{2}$ as positively directed away from $S$ and write $\left(8^{\prime}\right)$ and $\left(8^{\prime \prime}\right)$ in the form

$$
\begin{gathered}
\frac{d V}{d n_{1}}=\frac{d V}{d n_{2}}, \\
\frac{d V}{d n_{1}}+\frac{d V}{d n_{2}}=-4 \pi \sigma .
\end{gathered}
$$

The function $V(M)$ has a constant value, say 1 , on each surface element, and its derivatives are continuous as we approach an interior point of such an element [9, a, p. 165]. If then we define $v_{1}(M)$ locally as equal to $V$ on the side 1 of the surface element and as equal to $2-V$ on the side 2 of that element, the function $v_{1}(M)$ and its first partial derivatives will be continuous across the element, on account of (20); and $v_{1}(M)$ will be harmonic at points of the element. Consequently the surface element itself will be a piece of analytic surface, being a level surface, without singularities, of a harmonic function. Similarly an extension of $V$ from the side 2 to the side 1 yields a harmonic function $v_{2}$.

It may be shown that there exists a two-valued harmonic function $v(M)$ which has the following properties:

(a) Either branch of the function is harmonically extensible along any curve in space which does not meet $s$.

(b) The function is bounded and one of its two values tends to 0 at $\infty$, the other to 2 .

(c) If the two values $v_{1}(M), v_{2}(M)$ of $v$ are distinct, a closed path which starts from $M$ and loops $s$ once, returning to $M$, carries $v_{1}(M)$ into $v_{2}(M)$, and vice versa, whereas if the closed path does not loop $s$ it carries each value back in to itself.

(1) At every point $M$ not on $s, v_{1}(M)+v_{2}(M)=2$.

(m) Every point of $s$ is a limit point of the locus $v(M)=1$.

We obtain the function $v(M)$ by applying the Schwarz alternating process (in a form which is equivalent to the sweeping out process, 
without its distributions of mass) to a three-dimensional Riemann multiple space $\mathfrak{M}$. In our case the curve $s$ serves as a branch curve, analogous to a branch point in the complex plane, and the Riemann manifold may be constructed simply, by introducing the proper cut space. The necessary cut surface $\Sigma$ may be drawn, for example, as the image in terms of the transformation $\xi$ of a right circular cylindrical surface with base $\sigma$ (in the $x y$ plane) and extending to $\infty$ (in the direction of the positive $z$ axis). The function $v(M)$ is to be single valued in each of the cut spaces. The double cut space may again be replaced by a finite number of simple overlapping spaces by replacing the cylindrical surface by truncated conical surfaces within and outside it, and by means of these overlapping regions the connections of the Riemann manifold may be made across the cut surface.

At $\infty$ in the first sheet of $\mathfrak{M}$ within the half cylinder $\Sigma, v_{1}$ is to be assigned the value 0 and outside it the value 2, whereas in the second sheet $v_{2}$ is to be assigned the value 2 at $\infty$ within the cylinder and the value 0 at $\infty$ outside it; and the Schwarz process is employed to draw the two functions $v_{1}, v_{2}$ together through the simple regions which overlap the cuts. There are however one or two difficulties, to expound the details of which would extend unduly the length of this paper. In the first place, the curve $s$ is a curve of zero capacity and hence will not act as a carrier for boundary values, in spite of the fact that $v(M)$ is to assume the value 1 on $s$; and in the second place, the surface $\Sigma$ and the pseudo-conical surfaces are merely the topological images of smooth surfaces and are therefore not necessarily free of sets of points which are irregular for the Dirichlet problem.

The first of these difficulties is overcome by replacing $\sigma$ by a sequence of tori $\sigma_{n}$ condensing to $\sigma$ as $n \rightarrow \infty$. For each of the corresponding surfaces $\Sigma_{n}$ in $B$ a Riemann manifold $\mathfrak{M}_{n}$ is left, when the interior of $\Sigma_{n}$ is cut out, and a function $v^{(n)}(M)$ is set up which has the given properties at $\infty$ and corresponds to the value 1 on the boundary $\Sigma_{n}$. The second difficulty is handled by making use of the fact that the generalized Dirichlet problem is solvable for domains in which points are simply counted and by performing the analysis in terms of functions which are superharmonic on the interior of the whole Riemann manifold. It is proved that the successive alternate functions are superharmonic even at the exceptional points of the cross cuts which define the overlapping regions. ${ }^{4}$

4 Incidentally it may be noted that the above process for solving the generalized Dirichlet problem for a domain on a multiple space of a finite number of leaves enables one to arrive at the Green's function for the domain, which has at a given point a singularity of the form $1 / r$ on only one of the leaves. 
The sequence $\left\{v^{(n)}\right\}$ has a limit function $v(M)$ which is two-valued and of course harmonic in any simply counted bounded domain, so that it evidently satisfies (a). It is easily shown to satisfy (b) on account of the uniformity of convergence except in the neighborhood of $s$, and (c) and (l) similarly, since each function $v^{(n)}(M)$ has those properties. Because of the non-uniform convergence in the neighborhood of $s$ the property $(\mathrm{m})$ is established with a little more difficulty; it is proved by showing that in any neighborhood of a point of $s$ there lies for each $n$ at least one point $P_{n}$, distant from $s$ by more than a given positive $\delta$, where $v^{(n)}\left(P_{n}\right)=1$. The function $v(M)$ is not necéssarily continuous at $s$, for $s$ has not been restricted as to smoothness, but all of its points are limit points of the surface $S$ which is the locus $v(M)=1$. It is clear that $S$ occludes no points from $\infty$, that is, encloses no portion of space; in fact if the contrary were true we should have $v(M) \equiv 1$ in that portion, and therefore throughout space.

The facts just given about $v(M)$ lead to a more geometrical interpretation of the relation of $s$ to $S$. Any simple closed curve which loops $s$ once (having no points in common with $s$ ) has points in common with $S$. If in the neighborhood of $S$ the curve is analytic, and cuts $S$ at nonsingular points, it has an odd number of points in common with $S$, counting multiple intersections in the usual way.

We pass now to the converse statement. The two-valued harmonic function which satisfies the conditions (a), (b), (c) is unique.

The proof of this theorem lies essentially in an extension of Kellogg's uniqueness theorem, which may be stated as follows: Let $u(M)$ be harmonic and bounded on a domain $\Omega$ on a manifold $\mathfrak{M}$, which consists of a finite number of sheets, tending to 0 at $\infty$ if $\Omega$ is not a bounded domain, and let $m$ be the upper bound of $u(M)$, $m>0$. Let $F$ be the set of points $Q$ on the boundary of $\Omega$ where lim sup $u(M) \geqq m-\epsilon$ as $M$ tends to $Q$ on one or another leaf of $M$. Then $F$ considered as a set of points in ordinary space is of positive capacity. The details of the proof are not difficult, but must be omitted here on account of lack of space.

As a consequence of this theorem, since $s$ is of zero capacity, the difference of two functions which satisfy (a), (b), (c) will have to have its upper and lower bounds on $\mathfrak{M}$ both equal to zero.

The surface $S$, thus determined as the level surface $v=1$ of the function $v$, furnishes the minimum capacity among all surfaces $S^{\prime}$ which have the following properties: $S^{\prime}$ is bounded and every point of $s$ is a limit point of $S^{\prime}$; except in the neighborhood of $s, S^{\prime}$ consists of a finite number of regular elements sufficiently smooth for an application of Green's theorem; every closed curve which loops $s$ has a 
point in common with $S^{\prime}$. Thus $S^{\prime}$ is a cut surface for $\mathfrak{M}$. The proof depends upon a comparison of the energies of the conductor potential for $S$ and of the conductor potential for $S^{\prime}$ for the same total mass, in terms of Green's theorem-a comparison which is made possible by evaluating the Dirichlet integral for the conductor potential of $S$ first on $\mathfrak{M}$ and then on $S^{\prime}$. The proof has already been given in detail $[9, b]$.

6. Open surfaces of a finite number of sheets. The nature of our problem will be more clearly brought out by considering a slightly more general case, for example, the image $s_{1}, s_{2}$ in $B$ of two nonlinked circles $\sigma_{1}, \sigma_{2}$. In this case the connections of the Riemann manifold $\mathfrak{M}$ are easily imagined, since the branches of the two-valued function $v$ are to be interchanged by analytic extension along any circuit which links $s_{1}$ or $s_{2}$ once, and thus unchanged by a circuit which links them both. If we denote by $u^{(1)}, u^{(2)}$ the two functions $v^{(1)}-1, v^{(2)}-1$ where $v^{(1)}$ and $v^{(2)}$ are the two-valued harmonic functions belonging to $s_{1}, s_{2}$ separately, the product $u^{(1)} u^{(2)}$ will be single valued on $\mathfrak{M}$; and also the function $u(M)$, defined by the equation

$$
v(M)-1=u^{(1)}(M) u^{(2)}(M) u(M),
$$

will be single valued in ordinary space. By expressing the fact that the Laplacian of the left-hand member is zero, and utilizing the fact that $1-u(M)$ vanishes canonically as $M$ tends to $\infty$, and is represented by a potential, an integral equation may be found for the unknown function $u(M)$.

But it may also be remarked that if the curves $s_{1}, s_{2}$ are joined by a strip, arbitrarily narrow, so that $s$ is made a simple space curve in the manner of $\S 5$, the capacity in the new situation becomes a limiting case of that of the old, since the capacity of the strip may be made arbitrarily small. In other words, the problem of finding a single continuum, or even a single surface, in three dimensions which contains both $s_{1}$ and $s_{2}$ is illusory; for the capacity of a joining curve may be made zero. Hence our problem refers essentially to the determination of surfaces, finite in number, which will be cut by any circuit which loops a component part of $s$. In two dimensions there is, however, also a second problem analogous to our three-dimensional one.

We close by discussing qualitatively some simple cases. If the curve $s$ lies in a plane, the surface $S$ is the plane surface enclosed. Nevertheless the surface $S$ in general is evidently not a minimal surface. In fact, if $s$ consists of two circles $s_{1}, s_{2}$ which are parallel cross sections of a long circular cylinder, at a considerable distance from each other, 
the minimal surface would consist of the two parallel circular areas. On the other hand, it is evident that the equation (8) is not thus satisfied, so that the two flat discs do not constitute the arrangement for minimum capacity.

In the case of the Plateau problem, if the two circles just described are brought closer together, the discs remain a solution until there is actual contact; on the other hand, if the circles are withdrawn while they are bridged by the constricted longitudinal surface (the catenary of revolution) which satisfies the condition of zero mean curvature, this surface maintains itself until a central node is reached by means of the constriction. With the present problem there is only one surface at any stage, the unstable situation being avoided, in the continuous deformation, by the bulges in the disc-shaped surfaces which are required to minimize the energy. These are caused by the action at a distance which is expressed in terms of (8).

It is seen also by means of (8) that the surface $S$ lies within the closed convex cover of $s$.

If the two circles of the previous paragraphs are connected along elements of the cylinder by means of a narrow strip, so that $s$ becomes a simple curve, and the strip is twisted so that one of the circles is overturned, we have another figure which changes its character markedly as the circles are brought together. In fact, the surface changes continuously from a two-sided one when the circles are far apart, into a nodal surface and then finally into what is evidently a one-sided one when the circles are sufficiently close so that the curve $s$ can be the boundary of a narrow Möbius strip.

The author must admit, in conclusion, that the methods described in this three-dimensional discussion are quite specialized, and it is hard to see how they can be used in a more general class of problems in the calculus of variations. Perhaps the introduction of a vector potential would offer some advantage for the general point of view.

\section{REFERENCES}

1. H. Poincaré, Sur un théorème de $M$. Liapounoff, relatif à l'équilibre d'une masse fuide, Comptes Rendus de l'Académie des Sciences, vol. 104 (1887), pp. 622-625; also in Figures d'Équilibre d'une Masse Fluide, Paris, 1902.

2. A. Liapounoff, Sur la stabilitế des figures ellipsoidales d'équilibre, Thèse, 1884, translation, Annales de la Faculté des Sciences de Toulouse, vol. 6 (1904), pp. 5-116.

3. G. Faber, Beweis, dass unter allen homogenen Membranen von gleicher Fläche und gleicher Spannung die kreisförmige den tiefsten Grundton hat, Bayerische Akademie der Wissenschaften, 1923, pp. 169-172.

4. G. Pólya, Betrag zur Verallgemeinerung des Verzerrungssatzes auf mehrfach zusammenhängende Gebiete, Akademie der Wissenschaften, Berlin, 1928, Part 2, pp. 228-232 and pp. 280-282; 1929, Part 2, pp. 55-62. 
5. G. Szegö, Über einige Extremalaufgaben der Potentialtheorie, Mathematische Zeitschrift, vol. 31 (1929-1930), pp. 583-593.

6. O. D. Kellogg, (a) Foundations of Potential Theory, Berlin, 1929.

(b) Singular manifolds among those of an analytic family, this Bulletin, vol. 35 (1929), pp. 711-716.

7. E. Landau, Über die Blochsche Konstante und zwei verwandte Weltkonstanten, Mathematische Zeitschrift, vol. 30 (1929), pp. 608-634.

8. R. M. Robinson, The Bloch constant for a schlicht function, this Bulletin, vol. 41 (1935), pp. 535-540.

9. H. Grötsch, (a) Über konforme Abbildung unendlich vielfach zusammenhängende schlichter Bereiche mit endlich vielen Haufungsrandkomponenten, Berichte, Sächsische Akademie der Wissenschaften, Mathematische-Physikalische Klasse, vol. 81 (1929), pp. 51-86.

(b) Über ein Variationsproblem der konformen Abbildung, ibid., vol. 82 (1930), pp. 251-263.

10. G. C. Evans, (a) Surfaces of minimal capacity, Proceedings of the National Academy of Sciences, vol. 26 (1940), pp. 489-491.

(b) Surfaces of minimum capacity, ibid., pp. 664-667.

University of California 